\title{
FACTORES MOTIVACIONALES POSITIVOS Y NEGATIVOS EN LOS TRASTORNOS DE PERSONALIDAD
}

\author{
V. Pelechano \\ A. Pastor \\ Universidad de La Laguna (Tenerite)
}

\section{RESUMEN}

793 personas han cumplimentado la escala de Loranger sobre trastornos de personalidad y diversos cuestionarios sobre factores contextuales de personalidad relacionados con el mundo laboral. El análisis de los coeficientes de correlación arroja resultados altos y muy altos entre la mayoría de trastornos y factores motivacionales. El estudio de las diferencias en motivación por volumen de trastornos sugiere la existencia de cuatro grupos de factores de motivación que actúan de manera diferenciada. El análisis de las diferencias entre trastomos y motivación presenta resultados que pueden ayudar al diagnóstico diferencial y evitar algunas de las solapaciones. Los resultados demuestran que los factores contextuales de personalidad desempeñan un papel importante en la evaluación de los trastomos de personalidad.

Palabras clave: TRASTORNOS DE PERSONALIDAD Y MOTVACIÓN, VALOR DIFERENCIAL INTRA-TRASTORNOS DE FACTORES MOTIVACIONALES.

Una primera versión de este trabajo se presentó en el 9th European Congress in Psychology, Granada, 2005.

Correspondencia: vpelecha ull.es 


\section{SUMMARY}

793 Spanish adults (18-64 years old) have fulfilled diverse questionnaires (personality disorders by Loranger and 4 scales related to motivational working factors thorough 11 factors). Correlation analysis shows high and very high coefficients between personality disorders and motivational factors. Differential analysis among groups offer four types of motivational factors engaged to personality disorders with linear and non-linear relationships between personality disorders and motivational factors. The differential analysis according to possible existence of a personality disorder permits to isolate differences among motivation factors related with personality disorders. These differences can be helpful in order to isolate specificity in personality disorders.

Key words: PERSONALITY DISORDERS AND MOTIVATION, DIFFERENCES AMONG PERSONALITY DISORDERS IN FUNCTION OF MOTIVATIONAL FACTORS.

\section{LA INCLUSIÓN DE LA MOTIVACIÓN EN LA PERSONALIDAD Y EN LOS TRASTORNOS DE PERSONALIDAD}

Aunque la motivación humana representa una parte sustancial de la personalidad, cuestiones administrativas y de organización de la enseñanza han ayudado a su separación académica en materias distintas, que "pertenecen" a departamentos distintos en España y han promovido la idea de que se trata de aspectos muy diferentes entre sí, lo que no ayuda, en especial, ni a la formación ni al desarrollo de la psicología como ciencia, ni como profesión. Dentro de la psicología de la personalidad existen teorías que, como la de R. B. Cattell, incluye un gran apartado sobre análisis motivacional y otras, como la de $\mathrm{H}$. J. Eysenck que no la tematiza. En ambos casos les asiste parte de razón: en la medida en que se asimile la motivación al estudio de la dinámica personal, debería poder ser incorporada a la psicología de la personalidad y en especial, en el apartado que estudia la etiología de la acción, de los cambios en conducta y experiencia interna. 
Pero, a la vez, en la medida en que el estudio de la estructura de la personalidad lleva consigo un estudio intenso acerca de la gestación de esa estructura, su cambio y en definitiva, el estudio de los determinantes de esa estructura, resulta muy difícil de mantener la motivación como algo distinto y con suficiente entidad como para desgajarla de la psicología de la personalidad. En la medida en que la introversión, por ejemplo, se defina como una patrón complejo de acciones que se acompaña de elementos vivenciales concretos y con una bașe biológica claramente identificada (ritmos de activacióninhibición cortical), parece claro que la introversión sería tanto una "dimensión" de la personalidad como un elemento "etiológico" de la acción y del pensamiento, esto es, un elemento "motivacional".

Desde el modelo de parámetros, que el autor seniorde este trabajo viene defendiendo desde hace más de treinta años (Pelechano, 1973, 1975, 1997, 2000; Pelechano y Servando, 2004) se puede encontrar una solución a este tipo de problemas. La idea básica parte de los niveles de consolidación y de generalidad de los elementos constitutivos (rasgos, dimensiones) que forman la personalidad. Se distinguen usualmente, al menos tres niveles de consolidación-generalización de atributos de personalidad: un primer nivel "básico" en el que se incluyen las dimensiones de corte temperamental así como las socioactitudinales y básicas comprometidas con valores (sirva como ejemplo la escala usual de extraversión y de neuroticismo de $\mathrm{H}$. J. Eysenck).

Un segundo nivel cubre los atributos de personalidad que se desarrollan y poseen su mayor relevancia en los distintos contextos de vida tales como la familia, el mundo laboral o el mundo de las distracciones y agrupaciones de consolación/ayuda social: la motivación de logro, reacciones de afrontamiento directo ante situaciones de estrés, la afiliación o la intimidad se encuentran en este caso; se trata de atributos personales que se relacionan con acciones concretas en situaciones de vida que tienden a repetirse y en ellas poseen un gran poder comprensivo y predictivo. Teóricamente deberían "derivarse" de las básicas pero empíricamente no es así. En la actualidad existen relaciones complejas y empíricamente intrincadas entre estos dos niveles (véase un ejemplo en Pelechano, 2000) aunque pueden elaborarse pruebas que funcionen como "marcadores" de uno 
u otro nivel). Estos atributos son estables básicamente (aunque no de forma exclusiva) en la medida en que las condiciones de los contextos se mantengan estables, pero las relaciones con la conducta eficaz 0 ineficaz se modificarán si cambian estas condiciones contextuales. En buena cuenta, representarian maneras de entender la estabilidad y la inestabilidad en interacción con los contextos de vida. En este trabajo nos restringimos a factores contextual-motivacionales referidos al mundo laboral.

En tercer lugar se encuentran los "estados" o atributos de personalidad situacionales, que se modifican con el cambio de situación, son puntualmente relevantes $y$, en todo caso, progresivamente relevantes a medida que se incrementa la estructura y especificación de situaciones. Al igual que se apuntó en el caso anterior, las relaciones entre este nivel y los otros dos no es simplemente de "derivación lógica". Las relaciones entre los atributos de distinto nivel son complejas y apenas conocidas. Pero sabemos, por estudios realizados a lo largo de los últimos 30 años, que poseen gran poder de predicción en tareas concretas y estructuradas en situaciones de corta duración. Y son de carácter tanto cognitivo como emocional y hasta psicofisiológico.

Debe tenerse en cuenta que los distintos atributos de personalidad (neuroticismo, extraversión, hostilidad, etc.) se diferencian entre si, no solamente en cuanto a sus contenidos significativos, sino asimismo, respecto a su nivel de consolidación-generalización, de manera que "extraversión" o "neuroticismo" pueden ser evaluadas en uno u otro de los tres niveles. Asf, neuroticismo, tal y como se evalúa en las escalas de H. J. Eysenck es básico, pero la evaluación de la ansiedad de test o de rendimiento en contextos evaluativos sería neuroticismo contextual y el nerviosismo, temblores o pensamientos autodevaluativos referidos a situaciones concretas serían indicadores de neuroticismo-ansiedad situacional. Asimismo hay que decir que existen factores que favorecen el rendimiento y otros que lo perturban en todos los niveles. En el dominio motivacional-contextual, por ejemplo, la inhibición del rendimiento ante el estrés es un factor perturbador, mientras que la tendencia a la sobrecarga de trabajo, la exigencia personal y la búsqueda de perfeccionismo en la realización de tareas, se presentan como factores "positivos". Ambos tipos de factores pueden 
desempeñar papeles relevantes en los trastornos específicos de personalidad y este trabajo tiene como uno de sus objetivos poner a prueba esta posibilidad.

Cuando se definen los trastornos de personalidad, se identifican (DSM-IV-TR, 2000) como un patrón de experiencia interna y de conducta que se desvía de manera muy marcada de las expectativas de la cultura del individuo que lo presenta, se dice que debe encontrarse en el inicio de la fase adulta (o poco antes), es estable y comporta malestar o perjuicios para el sujeto. Se exige que exista un patrón anómalo en, al menos, dos de las siguientes áreas: (a) cognición (modo de percibir y de interpretarse a si mismo/a, a los demás o a los sucesos que le rodean); (b) afectividad (rango, intensidad, labilidad y adecuación de la respuesta emocional); (c) el funcionamiento interpersonal y (d) control de impulsos. Se manifiesta de forma inflexible en un amplio rango de situaciones personales y sociales de manera que promueve sufrimiento o discapacidad en algunas áreas funcionales relevantes y no se debe a otro trastorno mental, ni como consecuencia de acción de alguna sustancia, enfermedad física o traumatismo (por ejemplo, traumatismo craneoencefálico).

Por su parte, en la Clasificación Internacional de Enfermedades (CIE-10, 1992) se caracteriza a los trastornos de personalidad como expresión de un "estilo de vida y de la manera característica que el individuo tiene de relacionarse consigo mismo y con los demás", son duraderos y tienden a "abarcar aspectos múltiples de las funciones psicológicas y del comportamiento" (p. 247-8). Y, a la hora del diagnóstico: "el clínico ha de considerar todos los aspectos funcionales de la personalidad, aunque el diagnóstico, para ser simple y eficaz, se referirá sólo a aquellas dimensiones o rasgos en los que se alcancen los niveles de gravedad propuestos" ( $p .248$, el subrayado de textó es nuestro). En su sentido más preciso, la tarea diagnóstica sería excesivamente larga si se tuvieran que evaluar "todos los aspectos funcionales de la personalidad" $y$, en aras de la "utilidad" deberá restringirse solamente a las "dimensiones o rasgos" que se presenten como patológicos. En la especificación de dominios psicológicos a tomar en cuenta no se hace referencia a "toda" la personalidad sino que se restringirá a la parcela en la que se detecta falta de armonía y que afecte a varios aspectos de la personalidad "por ejemplo, a la 
afectividad, a la excitabilidad, al control de los impulsos, a las formas de percibir y de pensar y al estilo de relacionarse con los demás" (p.250), más adelante se refiere a que el comportamiento debe ser generalizado $a$ un "conjunto amplio de situaciones individuales y sociales" ( $p .250$ ). $O$, dicho con otras palabras: no se supone un deterioro o psicopatología en todos los sectores del funcionamiento del individuo, sino en algunos que sean relevantes; desde la perspectiva que se está defendiendo aquí, se trataría de elementos que aparecen de forma muy marcada en unos contextos más que en otros, y de ahí que el tipo de atributos personales que hemos llamado "contextuales" puedan desempeñar un gran valor diagnóstico, en la medida en que se adecuan más que los básicos a las situaciones de vida "en contexto".

Al margen de las características concretas de cada trastorno, sí está claro que se exige la presencia de perturbación del funcionamiento personal, que perdura a lo largo del tiempo, que no se explica por alguna causa "externa" al propio trastorno y que es invasiva en el sentido de que puede presentarse en uno o más contextos específicos de vida pero no necesariamente en todos. De hecho, existen personas que sufren de ciertos trastornos de personalidad que dificultad su vida en familia pero que los hace muy eficaces en el contexto laboral (por ejemplo, ciertos casos de trastorno de personalidad obsesivocompulsivo; como sucede asimismo con algunos casos de trastornos histriónicos y narcisistas). Desde aquí cabe pensar que son unos contextos de vida en los que se presentan los trastornos con mayor claridad y no en otros $y$, por ello, los atributos de personalidad que se encuentran activos en esos contextos pueden desempeñar un papel muy relevante en el diagnóstico y, posiblemente en el tratamiento de los trastornos de personalidad.

En este trabajo se realiza un estudio correlacional y diferencial entre los trastornos de personalidad identificados por la CIE-10 y un conjunto de factores de personalidad contextual-motiva-cionales, con el fin de poner a prueba la hipótesis general que subyace a todo el discurso: que los factores contextual-motivacionales de la personalidad deben desempeñar un papel relevante en los trastornos de personalidad.

En la medida en que todavía no es una práctica común el diagnóstico de los trastornos de personalidad en muchos servicios 
asistenciales, en el estudio ha participado una muestra de "población normal". Y se ha utilizado un cuestionario como rastreo de la posible existencia de trastornos de personalidad. Se asume que cerca del $10 \%$ de la población adulta sufre de uno o más trastornos de personalidad (Benjamin, 1996) y que entre la cuarta y la tercera parte de los casos que piden asistencia clínica por ansiedad o depresión, tiende a presentar, al menos, un trastorno de personalidad concomitante. El estudio de los codeterminantes de personalidad de estos trastornos puede permitir un mejor conocimiento de la funcionalidad que les caracterizan así como el diseño de tratamientos que sean más eficaces que los actualmente conocidos (lo que no resultaría muy difícil, de entrada, dada la carencia de tratamientos eficaces en la mayoría de trastornos identificados).

\section{MÉTODO}

\section{Particlpantes}

Un total de 793 adultos españoles ( $62,9 \%$ mujeres), entre 18 y 64 años de edad (16,52\% entre $18-24$ años; $56,87 \%$ entre $25-44$ años y $26,6 \%$ entre $45-64$ años). Poco más de la tercera parte, solteros $(35,1 \%)$ y un porcentaje similar de casados $(36,4 \%)$. La tasa de divorciados fue del $2,9 \%$.

Por lo que se refiere al nivel profesional, el 1,4\% estaban sin empleo; poco más de la mitad (51\%) eran trabajadores manuales; el 7,3\% eran administrativos, pequeños empresarios y profesionales de tipo medio, el $11,6 \%$ eran estudiantes universitarios, otro tanto de pensionistas $(11,1 \%)$ y el $3 \%$ eran titulados superiores y profesiones liberales.

\section{Pruebas}

Se han utilizado cuestionarios de dos tipos:

1. Cuestionario de trastornos. Se ha utilizado la escala de Loranger para los trastornos de la CIE-10 (Loranger, 1988; Loranger et al., 1997). Es una escala corta que permite rastrear la posible existencia de los trastornos específicos de personalidad que identifica la Organización Mundial de la Salud. 
2. Cuestionarios de personalidad. Se han utilizado distintos cuestionarios que evalúan el funcionamiento personal en el dominio laboral y en relaciones personales que fueron los siguientes:

- Cuestionario HO de Hostilidad (Pelechano, 2000c) que evalúa tres factores empíricos denominados: dureza de trato con logro de fines, malevolencia con recelo y rencor y desconfianza en el ser humano.

- Cuestionario R2 de rigidez laboral (Pelechano, 2000b), que está formado por dos factores reiteradamente encontrados desde hace más de tres décadas: hipervaloración del propio trabajo con intolerancia de debilidad por una parte y por otra, convencionalismo social con uso de especialistas para resolver cuestiones diff́ciles. Recoge la idea de exigencia rígida en el trabajo propio y aceptación de actitudes sociales básicas (más dinero, más trabajo) del funcionamiento social.

- Cuestionario MA de motivación laboral y reacciones ante situaciones de estrés (Pelechano, 1974). En sucesivos estudios ha aislado tres factores potentes que se denominan: imagen positiva y extremada de sí mismo/a, inhibición ante situaciones de estrés y motivación positiva ante estrés acompañado de sobrecarga y tendencia a la acción.

- Cuestionario LUCAM de locus de control, que se está aplicando desde hace dos décadas y que en las últimas factorizaciones ha repetido tres factores claros: control externo en resultados "negativos" con componentes depresivos, control bipolar (atribución personal de éxitos y exculpación de fracasos) y, finalmente, un factor de control social externo generalizado en el mundo laboral (básicamente en el rendimiento).

\section{Procedimiento de pase de pruebas}

Todos los participantes han sido colaboradores voluntarios. Los estudiantes han cumplimentado los cuestionarios en dos sesiones de pequeño grupo. El resto de participantes con procedimiento autoaplicado y remisión de pruebas pro correo o en sobre cerrado al Departamento de Personalidad, Evaluación y Tratamientos Psicológicos de la Universidad de La Laguna. La captación de los participantes no-estudiantes se hizo a través de los estudiantes y con estrategia de "bola de nieve". Se dio la opción de conocer los resultados 
y recibir un informe verbal acerca de puntuaciones individuales previa identificación, pasados tres meses de la cumplimentación de las pruebas.

Todos los participantes cumplimentaron la Escala de Loranger sobre trastornos de personalidad. Sin embargo, con el fin de no cansar a estos participantes y eliminar, en parte al menos, estereotipias de respuestas debido a cansancio o aburrimiento, los "paquetes" de factores de personalidad fueron cumplimentados por grupos entre 179 y 459 personas. No se puso un plazo fijo para la devolución del material, aunque se recogió entre 15 y 30 días después de su entrega.

Los datos así recogidos fueron analizados en un PC con el SPSS-11.

\section{RESULTADOS}

En la medida en que cada una de las escalas criteriales que forman el cuestionario de Loranger sobre trastornos de persnalidad presenta coeficientes de consistencia pobres, los resultados alcanzados entre las escalas de trastornos de personalidad y los factores contextuales de la personalidad tiende a ser baja. Con el fin de acercarnos a las "relaciones reales" entre los trastornos de personalidad y los factores de motivación empleados, una vez calculados los coeficientes de correlación, éstos han sido sometidos a cálculo de nuevo (corrección por atenuación) y éstos son los coeficientes que se manejan en el resto del trabajo.

\section{Un primer análisis sobre la relación entre factores motivacionales y trastornos de personalidad}

La tabla 1 recoge los coeficientes de correlación superiores a 0,30 entre los dos conjuntos de variables.

El número de participantes oscila entre 179 y 337 , lo cual no es un número despreciable. El $42,4 \%$ de los coeficientes encontrados son superiores a 0,40 , el $21,2 \%$ superiores a 0,50 y el $7,07 \%$ superiores a 0,69 , lo que significaría que cerca de la mitad de los coeficientes de los factores motivacionales comprometidos con el mundo laboral presenta correlaciones muy significativas con los trastornos de personalidad, por encima del $10 \%$ de varianza. 


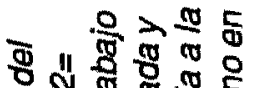
प $\frac{0}{0} \cdot \frac{\pi}{0}$

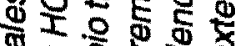

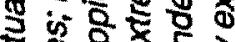
홍요원 농 $\frac{2}{0} \div$ 888 옹ำ

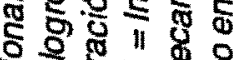
은

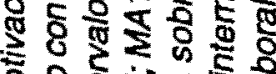
은 E 6 11 ต 58 눙

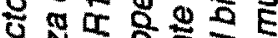

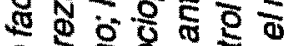
ㄴํㅇ

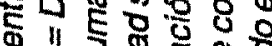
55 동ำ 응 ญैं के 응 5 궁 ชั ธิ Q $5 E$ is is

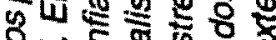

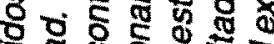
\%

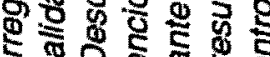
500 워

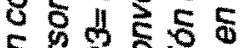
ㄷํㅇㅇㅝ क 5 पे

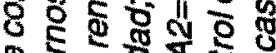
\%

응응

एह ᄃ $\leqslant 8 \%$

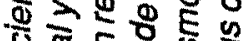
पू 5 . क 8 क ह

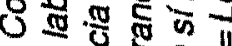
- 8 동 $\frac{11}{11}$

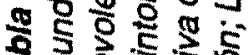

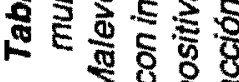

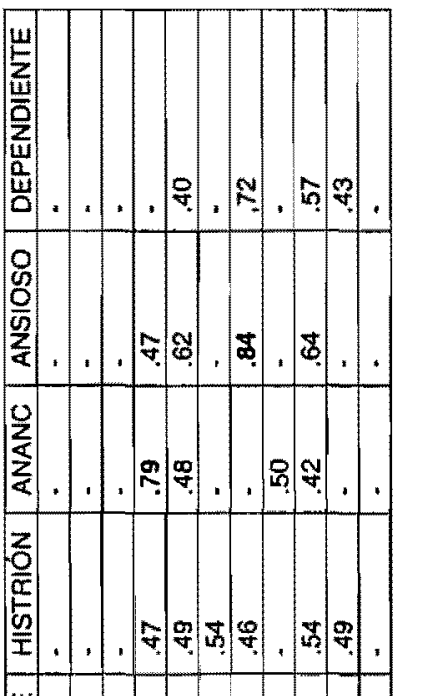

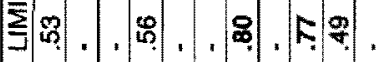

3

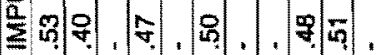

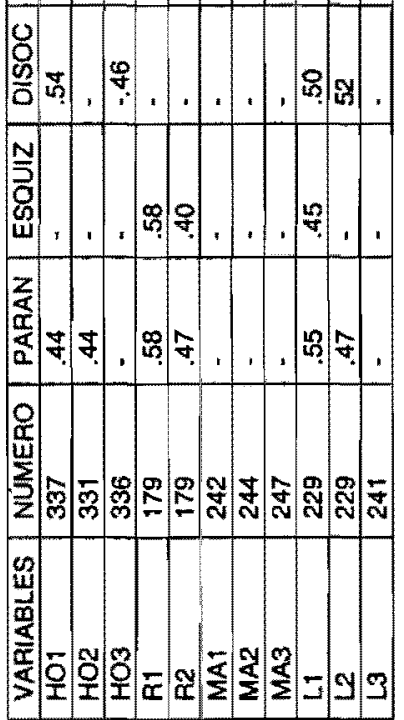

$\frac{\mathrm{g}}{\mathrm{g}}$

돋

음

总

으

$>$

응

g

है

ш

을

8

둥

응 등

으 흔

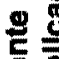

틍

$\frac{\text { a }}{9}$

동

릉 을

으 독

뜽 흥

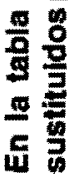


Por otro lado, solamente un factor de locus de control (externo y generalizado con componentes depresivos) presenta coeficientes significativos con todos los trastornos de personalidad. En dos de ellos, los coeficientes son superiores a 0,60: con el trastorno límite $(0,77)$ y con el trastorno ansioso $(0,64)$.

Un tercer punto de reflexión se refiere al signo. Solamente en un caso se ha obtenido un coeficiente negativo y se encuentra en el trastorno disocial, que comentamos más adelante, puesto que parece un tanto contra-intuitivo (desconfianza en el ser humano y trastorno disocial).

Un cuanto punto de reflexión genérico y probablemente, sería de esperar, es que el número menor de coeficientes superiores a 0,40 y, además, la media de coeficientes menos alta se encuentra localizado en el trastorno de personalidad esquizoide (3) y una tónica similar se encuentra en el anancástico y el trastorno de personalidad ansioso (4 coeficientes). $Y$ en estos casos, los factores son el ya mencionado locus externo generalizado y depresivo, junto con hipervaloración del propio trabajo (esquizoide, anancástico y ansioso) y el factor que hemos denominado convencionalismo y apelación a especialistas (esquizoide, anancástico y ansioso), lo que implicaría que en estos tres trastornos, los compromisos con el mundo motivacional laboral tienden a estar muy localizados. No deja de ser confirmatorio, que la relación más alta de entre todos los factores motivacionales en estos tres trastornos se encuentra entre el anancástico y el factor de hipervaloración del propio trabajo con intolerancia de la debilidad $(0,79)$. Por su parte, el trastorno ansioso presenta una relación muy alta con el factor de inhibición ante el estrés $(0,84)$, seguido de cerca por el factor de convencionalismo y apelación a especialistas $(0,62)$. EI trastorno dependiente, por su parte, presenta asimismo 4 coeficientes superiores a 0,40 y, asimismo, el factor de inhibición ante el estrés ofrece un coeficiente muy alto $(0,72)$.

El patrón que se observa respecto al trastorno disocial de la personalidad y motivación quizá merece un comentario especial. Hay que decir que el número de participantes que "supera" el criterio del número de respuestas requerido para el diagnóstico provisional del trastorno disocial es muy escaso y ninguno de ellos ha tenido problemas o detenciones de manera que se trata de un grado leve de "gravedad". En este sentido, parece más bien recoger a aquellas personas rebeldes 
y protestonas, más que de delincuentes antisociales violentos, que tienden a exculparse y no asumir muchas responsabilidades. Esto es lo que ofrece el patrón correlacional encontrado: dureza de trato con logro de fines al margen de los medios a usar en metas sociales cotidianas, atribución de control generalizado que, junto al coeficiente $(0,52)$ entre este trastorno y el locus de control bipolar, sugiere que, aunque no nos encontramos ante un psicópata-tipo, se trataría de personas egoístas, que se exculpan en los demás y que "dicen" que confían en el ser humano (tentativamente se podría aventurar que confían en los demás para cargarle de sus propias responsabilidades y para que les resuelvan los problemas). No deja de resultar ilustrativo que no aparecen factores motivacionales "positivos" tales como tendencia a sobrecarga de trabajo, gran exigencia personal en el trabajo hecho, ni convencionalismo - aceptación de normas - con posible apelación a los especialistas en el caso de encontrarse con problemas a los que no se ve solución a corto plazo.

Un último apartado cubre tres trastornos que presentan panoramas distintos entre sí. Se trata de los trastornos paranoide, impulsivo e histriónico. En todos ellos, más de la mitad de los coeficientes son superiores a 0,40 . Se trata de un grupo de trastornos que podrian caracterizarse por un acting out manifiesto. $O$, alternativamente, por ofrecer un panorama de notable "intensidad" psicológica en acción y reacción. El patrón correspondiente a paranoide e impulsivo es casi solapable, mientras que el histriónico se aleja un tanto de aquél. El trastorno paranoide y el impulsivo muestran relaciones similares ( $y$ de similar cuantía) con la dureza de trato y logro de fines al margen de los medios, malevolencia con recelo y rencor, hipervaloración del propio trabajo con intolerancia de la debilidad, locus de control externo generalizado y con componentes depresivos y control bipolar en éxitos $y$ fracasos. La diferencia entre ellos se encuentra en un compromiso entre el paranoide y el convencionalismo social, mientras que el impulsivo lo hace con la imagen positiva y extremada de sí mismo/a. El histriónico presenta similares resultados con los dos factores comentados de locus de control y en el factor de hipervaloración del propio trabajo, lo que es coherente con la definición básica del trastorno histriónico; a ello se añaden compromisos superiores a 0,40 en convencionalismo, imagen positiva y extremada de sí mismo/a como 
cabría esperar y una reacción de inhibición ante el estrés (entendido como demanda excesiva del trabajo que se pide, o del esfuerzo). Una nota final sobre estos tres trastornos es que los coeficientes obtenidos no llegan en ningún caso a 0,60 y 11 de los 18 no llegan a 0,50, por lo que cabe pensar que se trata de relaciones suaves aunque poco diferenciadas entre sl.

\section{Trastornos de personalidad y motivación laboral: todo tlpo de relaciones}

En un segundo momento se han agrupado los participantes en tres grupos: aquéllos que no alcanzaban la puntuación mínima para ser candidatos firmes a sufrir de uno o más trastornos de personalidad (se indican como grupo "0"); aquéllos que presentan puntuaciones escalares que son firmes candidatos a presentar uno u otro trastomo especifico de personalidad (representados por el grupo "1") $y_{\text {, }}$ finalmente, aquellos otros que alcanzaron puntuaciones que permitirian sospechar que poseen, al menos, dos trastornos de personalidad (representados por "2"). Posteriormente se han calculado las diferencias en medias (a partir de pruebas " $F$ " iniciales, complementadas posteriormente por pruebas " $t$ "). Los resultados se encuentran resumidos en la tabla 2 que pasamos a comentar.

Tabla 2. Resumen de diferencias estadísticamente significativas entre grupos formados por número de trastomos de personalidad existentes.

Comparaciones de diferencias en medias de grupos. La explicación en el texto

\begin{tabular}{|l|l|}
\hline 1.GRADACION POR GRAVEDAD & $0,1,2$ \\
-R1 (+): Hipervaloración trabajo propio, esfuerzo & $0,1,2$ \\
-MA2(-): Inhibición ante situaciones de estrés & $0,1,2$ \\
-L1(-): Locus externo depresivo en resultados & 0 y tener \\
\hline 2.NO PRESENCIAVPRESENCIA DE TRASTORNOS & y tener \\
-R2(+): Convencionalismo y acudir especialistas & no y $1-2$ \\
-HO3(-): Recelo y hostilidad & no y $0-1$ \\
3. NO LINEALES: NO TENER Y TENER MUCHO (O TENER & no y $0-1$ \\
POCO Y MUCHO & \\
-M1(+): Imagen positiva y extremada de sl mismo/a en & \\
trabajo & \\
-HO1(-): Dureza de trato y logro de fines & $2>0$ \\
-L2(-): Locus de control bipolar & $0>1$ \\
\hline 4. NO LINEALES: SOLO UNA DIFERENCIA & \\
-MA3(+): Acción ante estrés y sobrecarga & -L3(-): Locus externo en trabajo y relaciones personales \\
\hline
\end{tabular}


En la tabla se han agrupado las diferencias estadísticamente significativas encontradas en cuatro grupos. En el primer grupo, que se identifica como "gradación en gravedad", se insertan los factores que presentan relaciones lineales y directas entre motivación y trastornos de personalidad; en el segundo, que se identifica como "no presencia/presencia de trastornos se encuentran recogidas las diferencias que son de tipo "categorial"; en el tercer grupo se recogen resultados que sugieren relaciones no lineales ("no tener y tener mucho" o "tener poco y tener mucho") y en el cuarto grupo, finalmente, se encuentran los factores que, aun sugiriendo relaciones no lineales, presentan solamente una diferencia estadísticamente significativa. EI signo que se encuentra al lado de cada factor entre paréntesis significa si el factor es teóricamente facilitador o perturbador del rendimiento.

Lo primero que debe señalarse es que en cada uno de los grupos de tipos de relaciones se encuentran presentes factores motivacionales facilitadores y factores perturbadores del rendimiento y los resultados encontrados no siempre son interpretables de manera simple y directa.

Tres factores motivacionales presentan relaciones directas $y$ positivas con el volumen de trastornos: (a) crecimiento significativo en puntuaciones a medida que se incrementa el número de trastornos de personalidad en el factor de hipervaloración del propio esfuerzo con intolerancia de la incompetencia laboral, que es un factor que facilita el rendimiento laboral, aunque no las buenas relaciones personales; (b) asimismo, a mayor número de trastornos de personalidad, mayor inhibición ante el estrés (ansiedad contextual), que es un factor perturbador del rendimiento; (c) finalmente, asimismo a mayor volumen de trastornos de personalidad, mayor puntuación en el factor de control externo generalizado y depresivo. Debe tenerse presente que en estos trastornos, el correspondiente al disocial es muy escaso, como se ha comentado anteriormente en este trabajo.

Un segundo grupo de factores poseen un valor de "marcadores" en cuanto a existencia de trastorno, pero no gradúan su puntuación en función del número de los trastornos encontrados (tendrían una función "categorizadora"). Se incluyen aquí dos factores motivacionales, uno positivo (convencionalismo y acudir a especialistas para que resuelvan problemas) y otro negativo (recelo y hostilidad). En cierto sentido se pueden considerar como dos polos de un continuo (con oposición 
modal, que no contradictoria): en la parte positiva habría una actitud de defensa del convencionalismo en el funcionamiento laboral (por ejemplo, hay que trabajar más para ganar más) y en el polo negativo una desconfianza interpersonal y recelo en el trato humano. Estos dos elementos alcanzan una puntuación significativamente mayor cuando se da uno o más trastornos de personalidad frente a no presentar ringuno, pero no diferencian más allá en el sentido de que, frente a lo que sucedía en el apartado anterior, en este caso no hay diferencias entre tener uno o más trastornos específicos de personalidad.

El tercer grupo, formado por tres factores motivacionales, resulta una variante del anterior: las diferencias se detectan entre tener uno o dos trastornos (el factor teóricamente positivo de imagen positiva y extrema de sí mismo/a en el mundo laboral) pero no entre tener y no tener trastornos. Por otro lado, dos factores teóricamente perturbadores (dureza de trato y logro de fines al margen de los medios) y el factor de locus de control bipolar presentan diferencias solamente entre no tener trastornos y tener solamente uno.

El cuarto grupo, finalmente, recoge los factores motivacionales que poseen un valor de diferenciación aunque no se forma lineal clara. El factor motivacional positivo de tendencia a la acción en situaciones de estrés presenta puntuaciones estadísticamente significativas y mayores cuando se tienen dos o más trastornos frente a los que no presentan ninguno. El factor de locus de control externo en trabajo y relaciones personales (factor teóricamente negativo) presenta puntuaciones significativamente mayores entre no tener trastorno y tener uno, pero no diferencia entre tener uno o más de uno, ni no tener y tener más de uno.

\section{Diferencias no-contaminadas entre trastornos de personalidad (un apoyo para la especificidad de cada trastorno)}

Finalmente nos hemos acercado al problema de los concomitantes motivacionales de cada uno de los trastornos frente a los demás, con el fin de poder obtener información acerca de concomitantes de cada trastorno independientemente del resto. Para ello se han elegido las personas que cumplen con la existencia de un posible trastorno de 
personalidad según los criterios de corte que se asumen en la CIE-10 $y$ aislado los que poseerian ese trastorno y solamente ese. $Y$ en un segundo paso, se han comparado las puntuaciones en los distintos factores de motivación cumplimentados en este estudio entre cada trastorno de personalidad. Con ello tendríamos información acerca de lo que "diferenciaría motivacionalmente" a unos trastornos de otros. Y, secundariamente, la existencia de redundancias diferenciales (tanto en factores que diferencian entre factores específicos, como aquellos otros que no ofrecen patrones de diferenciación) al comparar distintos trastornos podría ser un marcador a tomar en cuenta a la hora de rastrear las solapaciones entre dimensiones de personalidad que se encuentran en los distintos trastornos (dicho con otras palabras, una explicación posible, aunque parcial, de la "comorbilidad" o coincidencia de más de un trastorno en una persona).

Los resultados se presentan resumidos y por lo que se refiere a tres trastornos, como ejemplos representativos (disocial, límite y dependiente). El signo " $<$ " 0 " $>$ " indica que la puntuación del "criterio" (trastorno disocial, límite y dependiente, respectivamente) en el factor motivacional respectivo, es menor o mayor que la puntuación en este factor en el otro trastorno. Los asteriscos se refieren al nivel de significación (uno, $p<0,05$; dos $p<0,01$ y tres $p<0,001$ ). Los resultados se encuentran resumidos en la tabla 3.

Tabla 3. Diferencias estadísticamente significativas en factores contextuallaborales entre distintos trastomos (existencia solamente de uno en cada caso

\section{(A).- Trastomo disocial frente a resto}

\begin{tabular}{|c|c|c|c|c|c|}
\hline TRASTOFNONARIAQLES & IMPULSIVO & UMTE & HSTRONOO & ANSIOSO & DEPENDIENTE \\
\hline 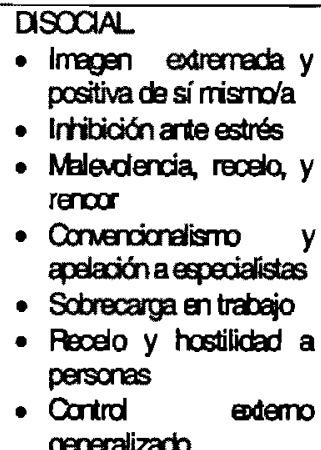 & $<$ & $<$ & $\begin{array}{l}< \\
< \\
<\end{array}$ & $<$ & 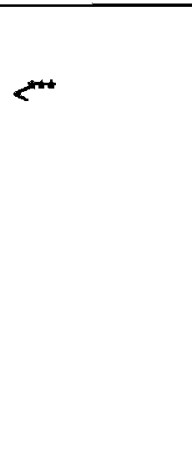 \\
\hline
\end{tabular}


(B). Trastorno límite frente a resto

\begin{tabular}{|c|c|c|c|c|c|c|c|}
\hline TRASTNAAAB & PAPAN & ESQUZ & DISOC & IMPUS & HSIRO & ANANC & ANSOSO \\
\hline 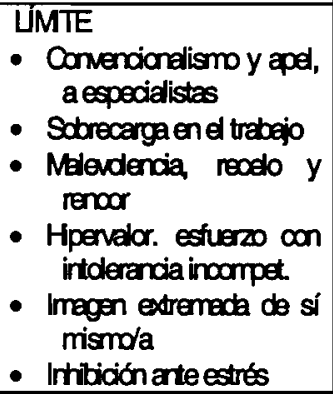 & $<$ & $<$ & $\begin{array}{l}>^{*} \\
>^{n+1}\end{array}$ & $\begin{array}{l}< \\
<\end{array}$ & $<$ & $>^{*}$ & $>$ \\
\hline
\end{tabular}

(C). Trastorno dependiente frente a resto

\begin{tabular}{|c|c|c|c|c|c|c|c|}
\hline TRASNARAB & PARAN & ESQUZ & DISOC & IMPUS & HSTRO & ANANC & ANSICSO \\
\hline 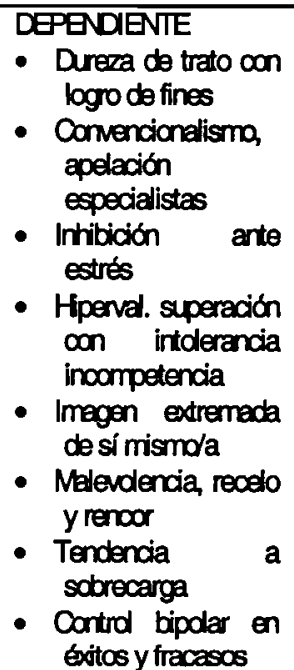 & $\begin{array}{l}< \\
> \\
>^{*} \\
>^{*}\end{array}$ & $>$ & $>^{*+*}$ & $\begin{array}{l}>^{*} \\
<^{*} \\
<^{*}\end{array}$ & $<$ & $\begin{array}{l}>^{+} \\
< \\
<\end{array}$ & $<*$ \\
\hline
\end{tabular}

Se ha elegido el trastorno disocial por la relevancia, pese a que en la muestra no existe un número alto que cumpla los criterios. El límite por su frecuencia creciente y el dependiente por la aparición en los últimos años de personas que se encuentran en esta condición y la escasez de tratamientos monográficos sobre él.

Por lo que se refiere al trastorno disocial hay que decir que en cinco de los 8 trastornos posibles se encuentran diferencias estadísticamente significativas en un factor: la inhibición ante el estrés presenta puntuaciones significativamente más bajas que en el trastomo 
disocial, en el impulsivo, limite, histriónico y dependiente, lo que sugeriria que esa reacción de inhibición, como parte de la inestabilidad emocional, sería diferenciadamente común.

Resulta de cierto interés que precisamente el trastorno histriónico es el que presenta mayor volumen de diferencias con el disocial, siendo asi que en la bibliografía se reconoce la dificultad de la diferenciación entre ambos; de acuerdo con las variables empleadas, el histriónico resulta significativamente con menos imagen extremada de sí mismo/ a $(p<0,05)$, menos inhibición ante estrés $(p<0,001)$, menos convencionalismo y apelación a especialistas $(p<0,05)$ y menos tendencia a sobrecarga en el trabajo $(p<0,01)$, por lo que las puntuaciones en estos factores podrían utilizarse como coadyuvantes en el diagnóstico diferencial.

El trastorno de personalidad ansioso presenta tres diferencias estadisticamente significativas respecto al disocial: más inhibición ante situaciones de estrés $(p<0,001)$, más recelo y hostilidad hacia las personas $(p<0,05)$ y menos control externo generalizado $(p<0,05)$. Posiblemente los dos primeros resultados sean atribuibles a la ansiedad, mayor en el trastorno ansioso que en el disocial.

El trastorno impulsivo presenta puntuaciones significativamente mayores que el trastorno disocial en inhibición ante estrés $(p<0,05)$ y en imagen extremada y positiva de si mismo/a $(p<0,01)$.

El trastorno límite ofrece puntuaciones significativamente mayores que el disocial en la inhibición ante situaciones de estrés $(p<0,001)$ y en malevolencia, recelo y rencor $(p<0,01)$.

Finalmente, el trastorno dependiente limita su diferencia a la inhibición ante situaciones de estrés $(p<0,001)$.

Las comparaciones, tomando como criterio el trastorno límite se presentan en 7 de los 8 posibles trastomos (el único trastomo "común" sin diferencias estadísticamente significativas con el limite sería el dependiente). Frente a lo que sucedía en el caso del disocial, aqui no existe un factor motivacional que presente diferencias significativas en todos los trastornos. Más arriba se han presentado las diferencias con el disocial por lo que no se repetirán aquí.

El trastorno impulsivo presenta diferencias estadísticamente significativas con el límite en tres factores: el límite se presenta con menos malevolencia y rencor $(p<0,001)$, menos hiper-valoración del 
esfuerzo y propio trabajo con intolerancia de incompetencia $(p<0,05)$ y menos imagen extremada de sí mismo/a $(p<0,05)$.

El trastorno ansioso presenta dos diferencias estadísticamente significativas con el límite: más convencionalismo y apelación a especialistas $(p<0,05)$ y más imagen extremada de si mismo/a $(p<0,05)$.

Existen cuatro trastornos específicos que presentan tan solo una diferencia estadisticamente significativa con el trastorno límite y que es específica para cada trastorno. Asi, el paranoide se presenta como más defensor del convencionalismo social $(p<0,05)$, el esquizoide con mayor tendencia en el factor de sobrecarga en el trabajo $(p<0,05)$, el histriónico mayor imagen extremada de sí mismo/a $(p<0,05)$ y el anancástico menos valorador del esfuerzo y menos intolerante de la incompetencia $(p<0,05)$. Esta "carencia" de especificidad del trastorno límite sería una explicación acerca de la dificultad de su análisis y tratamiento en la medida en que posee muchas características "normales" que se encuentran en los otros trastornos tambien y las diferencias encontradas no son muy grandes, cuando las hay, en los criterios de motivación laboral.

Finalmente, el trastomo dependiente. Como sucedia con el trastomo límite, no existe un factor que presente diferencias en todos los trastornos, pese a que se detectan diferencias estadisticamente significativas en siete de las ocho comparaciones posibles de trastornos. La diferencia con el disocial ya se ha comentado más arriba, por lo que no se repetirá aquí.

El mayor volumen de diferencias lo presenta con el trastorno impulsivo: el trastorno dependiente se presenta más inhibido ante el estrés $(p<0,05)$, valora menos la superación en el trabajo $(p<0,05)$, posee menos imagen extremada de sí mismo/a $(p<0,01)$ y muestra menos malevolencia y rencor $(p<0,05)$.

Dos trastornos ofrecen tres diferencias significativas aunque ninguna es redundante: con el trastomo paranoide y con el anancástico. El trastorno dependiente se presenta, frente al paranoide, con menos dureza de trato $(p<0,05)$, más convencionalismo $(p<0,05)$ y mayor inhibición ante situaciones de estrés $(p<0,001)$.

El trastorno dependiente frente al anancástico se muestra con mayor hipervaloración verbal de la superación $(p<0,001)$, menos 
imagen extremada de si mismo $(p<0,05)$ y menos malevolencia, recelo $y$ rencor $(p<0,05)$.

Existen dos diferencias estadísticamente significativas encontradas entre el dependiente y el histriónico: el dependiente se muestra con menos imagen extremada de si mismo/a que el histriónico y menos malevolencia, recelo y rencor $(p<0,05)$.

Otros dos trastornos de personalidad presentan cada uno una diferencia tan solo estadísticamente significativa: el trastorno dependiente muestra una mayor tendencia a la inhibición ante situaciones de estrés $(p<0,001)$ que el esquizoide y menos locus de control bipolar $(p<0,01)$ que el ansioso.

Estos resultados son coherentes con la definición teórica de los trastornos, por un lado, y por otro, ayudan a especificar unos trastornos frente a otros. De lo que podría deducirse que los factores motivacionales relacionados con el contexto laboral que hemos utilizado tienden a presentar patones específicos de diferencias, más en uso trastornos que en otros pero cuya utilización ayuda a esclarecer el espectro y correlatos de la sigrificación psicológica de los trastornos de personalidad.

\section{CONCLUSIONES Y DISCUSIÓN}

La primera conclusión es que los resultados alcanzados apuntan a la posibilidad y hasta conveniencia de modelos cuantitavos en el estudio de los trastornos de personalidad y, de rechazo, la demostración de que es posible y iluminador estudiar las relaciones entre los trastornos de personalidad y la psicología de la personalidad siempre que se entienda ésta con modelos multinivel de factores en función de su nivel de consolidación-generalización.

Como ejemplo de este punto de vista que defiende el modelo de parámetros desde hace más de tres décadas, el estudio de las relaciones entre trastornos de personalidad y factores motivacionales comprometidos con el mundo laboral ha arrojado coeficientes

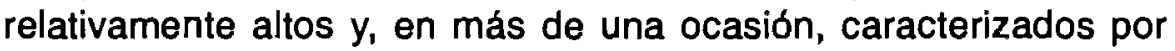
cierta inespecificidad, en todos o en grupos de trastornos. Una sugerencia que se puede derivar de ello es que estos factores de personalidad formarian un sustrato sobre el que se "identifican" los 
sintomas criteriales de los trastornos. En la medida en que los coeficientes de los mismos factores se encuentran y en cuantía similar, con distintos trastornos, ello explicaría la "redundancia" o solapación que se encuentra usualmente entre los distintos diagnósticos de trastornos de personalidad especificos: Ia insistencia por parte de un número progresivamente mayor de autores que, como Costelo (1996) y Benjamín (1996), desde perspectivas teóricas muy distintas, defienden el estudio de los atributos de personalidad más que de los "síntomas" de los trastornos se presenta como una posibilidad que al "ofrecer" mecanismos funcionales, ayudarian a eliminar parte de estas redundancias o, de rechazo, a replantear la existencia del número propuesto de trastornos específicos de personalidad y de esa manera.

Un segundo elemento a considerar es que, por lo que se refiere a los factores motivacionales empleados, los últimos 20 años han demostrado que se trata de factores "favorecedores" unos y "perturbadores" otros del rendimiento laboral. En los resultados se ha demostrado que se encuentran presentes estos dos tipos de factores en los trastornos específicos de personalidad y, por ello, la posibilidad de promover los "positivos" en la medida en que vayan aparejados no solamente con una independencia socio-personal, sino además, que suelen ir acompañados de sensación de bienestar personal. El hecho que en unos trastornos todo ello se acompañe de reacciones de inhibición ante el estrés sugiere que a veces puede ser converiiente y en otras no, la recomendación de "laborterapia"; en todo caso, el empleo de aspectos funcionales "positivos" ilustra que en el tratamiento de algunos de estos trastornos pueden incluirse elementos relacionados con esa eficacia laboral.

Un tercer aspecto a tomar en consideración se refiere a que existen unos factores con poder de diferenciación intra-trastornos y otros no. Que los factores motivacionales ofrecen, en muchos casos unos patrones de diferenciación que no se repiten y que enriquecen la significación psicológica y personal de los trastomos de personalidad, por lo que pueden utilizarse como elementos diagnósticos y, a partir de las relaciones que presentan estos factores con la conducta eficaz, como coadyuvantes en el diseño de tratamientos más eficaces. De los factores contextual-motivacionales que se han empleado en este 
estudio, dos se han presentado con mayor frecuencia: la imagen extremada de sí mismo valorativa del mundo laboral en concreto y la inhibición ante situaciones de estrés: se han presentado como elementos relevantes tanto en cuanto a compromisos con distintos trastornos, como a factores de diferenciación inter- trastornos. Mientras el primero tendría que ver con criterios de intensidad psicológica, el segundo es un representante del factor básico de ansiedad, en el contexto laboral. La imagen del yo en ambientes laborales se ha presentado como un elemento importante que no ha sido tomado en consideración en los acercamientos científicos y no psicoanalíticos al estudio de los trastornos de personalidad y pensamos que podría ser un elemento a tomar en cuenta tanto en lo que se refiere a diagnóstico como en paquetes de tratamiento.

En definitiva, los autores son conscientes de las numerosas limitaciones que posee un trabajo como el que hemos realizado, pero con todo pensamos que ofrece procedimientos de anáklisis, resuitados e ideas que pueden ser continuados y puestos a prueba en otros trabajos. $Y$, desde luego, el "alejamiento" de los trastornos de personalidad de la psicología científica de la personalidad parece más un empecinamiento de algunos grupos que una realidad deseable.

\section{REFERENCIAS BIBLIOGRÁFICAS}

Benjamín, L. S. (1996).- Interpersonal diagnosis and treatment of personality disorders, $2^{\text {nd }}$ edit., New York, The Guilford Press.

Costa Jr., J. P. T. \& McCrea, (eds) (2003).- The big five and personality disorders, Washington, American Psychological Association.

Costelo, C. G. (ed.)(1996).- Personality characteristics of the personality disordered, New York, John Wiley \& Sons.

De Miguel, A. \& Pelechano, V. (2000).- Estructura de personalidad y trastornos de personalidad: correlatos de personalidad de los trastornos de personalidad definidos pro clasificación de consenso, Psicologemas, 14, monográfico, 1-210.

Loranger, A. W. (1988).- Personality disorder Examination (PDE), Yonkers, DV Comunications.

Loranger, A. W., Janca, A., \& Sartorius, N. (eds)(1997).- Assessment and Diagnosis of Personality Disorder. The ICD-10 International 
Personality disorder Examination (IPDE), Cambridge, Cambridge University Press.

Organización Mundial de la Salud (1992).- CIE-10. Trastomos mentales y del comportamiento, Madrid, Meditor.

Paris,, J. (1998).- Working with traits. Psychotherapy of personality disorders, Northvale, NJ, Jason Aronson Inc.

Pelechano, V. (1997).- Una introducción al modelo de parámetros en psicología. En V. Pelechano (dir).- Psicología de la personalidad. I. Teorías, Barcelona, Ariel.

Pelechano, V. (2000a).- Psicología sistemática de la personalidad, Barcelona, Ariel.

Pelechano, V. (2000b).- Cuestionario R2 de rigidez: teoría mínima, composición factorial y datos de validez interna, Análisis $y$ Modificación de Conducta, 26, 469-508

Pelechano, V. (2000c).- Cuestionario de hostilidad y malevolencia, Análisis y Modificación de Conducta, 26, 779-817.

Pelechano, V. y Maestro, A. (en prensa).- Neuroticismo y trastornos de personalidad, Análisis y Modificación de Conducta, 31.

Pelechano, V. y Servando, M. A. (2004).- ¿Qué es la personalidad?, Madrid, Biblioteca Nueva. 